$\underline{\text { Research article }}$

\title{
Detection of Biofilm formation in device associated clinical bacterial isolates in cancer patients
}

\author{
FM Patel ${ }^{1}$, PN Goswami ${ }^{1}$, R Khara ${ }^{1}$ \\ Sri Lankan Journal of Infectious Diseases 2016 Vol.6 (1):43-50 \\ DOI: http://dx.doi.org/10.4038/sljid.v6i1.8086
}

\begin{abstract}
Introduction:

Biofilm is a community of bacteria that are attached to a substratum or surface. Bacteria in biofilm are embedded in extracellular polymeric matrix produced by the bacteria. Biofilm formation on indwelling medical devices such as catheters pose a critical medical problem. Both Gram-negative and Gram-positive bacteria can form biofilms on indwelling medical devices.
\end{abstract}

\section{Objectives:}

To determine the potential relationship between colonization of different medical devices by various clinical bacterial isolates, to determine the differences in biofilm formation in different conditions and to determine the minimum time and conditions necessary for the development of a homogenous and mature biofilm layer.

\section{Methods:}

The study sample consisted of 50 clinical strains isolated from different medical devices and another 50 strains (control group) isolated from swabs taken from peripheral and central catheter insertion sites. All isolates were characterized to species level using commercial identification cards (GN ID, GP ID; Biomerieux, France) on an automated ID AST system. Biofilm formation and quantification of activity was assessed using a microtitre plate assay.

\section{Results:}

Of the 50 clinical isolates, 42 were biofilm producers and 5 of the 50 surface swab isolates were weak biofilm producers. Of the 42 clinical isolates which produced biofilms, 24 were strong producers, 15 were moderate and 3 were weak producers. Catheter blood yielded the highest number (19/50) of biofilm producers. Acinetobacter spp. (15/50), Klebsiella pneumoniae (11/50), Pseudomonas aeruginosa (8/50), Staphylococcus spp. (7/50), and E. coli (6/50) were the most common isolates. Twenty hours incubation time was found to be optimum for detection of biofilms produced by bacteria. Concentration of glucose also played an important role in formation of biofilm with more biofilm formation in $0.25 \%$ glucose in tryptic soya broth.

Conclusion: The current study shows that biofilm producing Gram negative bacilli are present in vascular catheters. Methods that detect and quantify biofilms on the inner (luminal) as well as outer surfaces of catheters will provide the only true picture of biofilm colonization.

${ }^{1}$ The Gujarat Cancer \& Research Institute, Ahmedabad, Gujarat, India Address for correspondence: Dr Foram Patel, The Gujarat Cancer \& Research Institute, Ahmedabad, Gujarat, India Telephone +919898070240 Email :drforam9@gmail.com 
Keywords: Biofilm, device associated, cancer patient

\section{Introduction}

Biofilm is a community of bacteria attached to a substratum or surface. Bacteria in biofilm are embedded in an extracellular polymeric matrix produced by the bacteria. ${ }^{1}$ Biofilm formation on indwelling medical devices such as catheters pose a critical medical problem. Both Gram negative and Gram positive bacteria can form biofilms on indwelling medical devices. The most common biofilm forming bacteria are Enterococcus faecalis, Staphylococcus aureus, $S$. epidermidis, viridans streptococci, Escherichia coli, Klebsiella pneumoniae, Acinetobacter baumannii and Pseudomonas aeruginosa. ${ }^{2}$

Biofilm formation is a well-known pathogenic mechanism in device related infections in hospitals. Bacterial cells can detach from mature biofilms and spread to other organ systems. ${ }^{3,4}$ As a result, biofilms become sources of persistent and chronic infections. Bacteria in biofilms behave differently from planktonic bacteria, especially in terms of their response to antibiotics. ${ }^{2}$ Biofilm associated bacteria are often highly resistant to antibiotics. In addition, the complicated structure of biofilm with extracellular polymeric matrix can prevent antibiotics from reaching the bacteria. Bacteria in biofilms could also adopt a slow growing or starved state due to the altered microenvironment such as depletion of nutrition and accumulation of waste. The changed physiological state of bacteria could make them more resistant to antibiotics which target more active cell processes. ${ }^{5-8}$

When an indwelling medical device is contaminated with microorganisms, several variables determine whether a biofilm develops. First, the microorganisms must adhere to the exposed surfaces of the device long enough to become irreversibly attached. Once these cells irreversibly attach and produce extracellular polysaccharides to develop a biofilm, its rate of growth is influenced by flow rate, nutrient composition of the medium, antimicrobial drug concentration and ambient temperature. ${ }^{2}$. Apart from the temperature, previous studies have also shown the effects of nutrient composition and duration of incubation. ${ }^{9}$

\section{Objectives:}

- To isolate and identify Gram positive and Gram negative bacteria from patients with different indwelling medical devices.

- To study the isolates for quantitative in-vitro biofilm formation by giving them different growth condition and incubation times

\section{Methodology}

This study was carried out from February to September 2013. During this period, 326 different indwelling medical devices and blood and urine collected through an indwelling catheter were received in the microbiology laboratory. Positive cultures were obtained from 50 of these 326 samples. Peripheral vein sampling was done to rule out general bacteremia in all these patients to rule out seeding of the catheter through the blood. All isolates were characterized to species level 
using identification cards (GN ID,GP ID;Biomerieux, France) on an automated ID AST system (Vitek 2 compact, Biomerieux, France). The isolates were then tested for in vitro production of biofilm using a microtitre plate assay. ${ }^{9}$

Biofilm-producing reference strains of $A$. baumannii (ATCC19606) and P. aeruginosa (ATCC 27853) were used as positive controls ${ }^{10}$ and non-biofilm forming reference strains of $S$. aureus (ATCC25923) and E. coli (ATCC25922) as negative controls. ${ }^{11}$

A total of 50 strains isolated from swabs taken around the catheter insertion site were included in the control group.

\section{Biofilm formation and quantification of activity using a microtitre plate assay}

Three different types of liquid media i.e. tryptic soya broth, tryptic soya broth with $0.25 \%$ glucose and tryptic soya broth with $0.5 \%$ glucose were used. Isolated colonies were inoculated into each of these media and incubated for 16, 20 and 24 hours separately following which they were diluted 1:200 using the same media.

\section{Microtitre plate assay}

Biofilm formation was induced in 96-well flat-bottomed polystyrene microtitre plates. An aliquot of $200 \mu \mathrm{l}$ of diluted bacterial suspension was added to each well and incubated for 16, 20, and 24 hours at $37^{\circ} \mathrm{C}$. At the end of the incubation period, the well was carefully aspirated and washed twice with $300 \mu \mathrm{l}$ of phosphate-buffered saline (PBS, pH 7.2) to remove planktonic bacteria. Plates were kept for drying for $15 \mathrm{mins}$ at room temperature $\left(25^{\circ} \mathrm{C}\right)$.

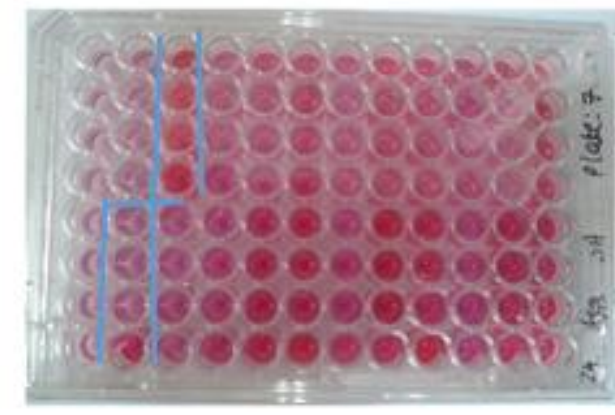

Fig: 1

Micro titre plate assay showing biofilm formation in Gram negative bacilli

$$
\begin{aligned}
& \mathrm{RC}-\text { reagent controls } \\
& \mathrm{NC} \text {-negative controls } \\
& \mathrm{PC} \text { - positive controls } \\
& \text { Remaining wells - samples }
\end{aligned}
$$

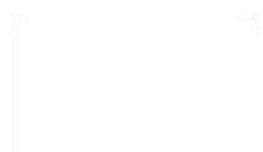

$\square$

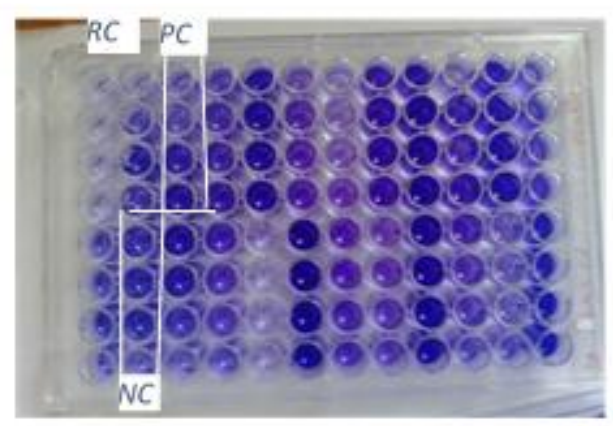

Fig: 2

Micro titre plate assay showing biofilm formation in Gram positive cocci

Biomass quantification of the biofilms was performed by staining as follows: $200 \mu 1$ of $0.1 \%$ safranin and $0.1 \%$ crystal violet was added to each well and left for 45 mins at room temperature. After incubation, the wells were carefully washed twice with distilled water to remove excess stain using an automated washer. $200 \mu \mathrm{l}$ ethanol/acetone (90:10) was then added to each well to dissolve the remaining stain from the wells (Figs $1 \& 2$ ). The optical density was then recorded at $492 \mathrm{~nm}$ with $630 \mathrm{~nm}$ reference filter using an ELISA reader(/DVQ ELISA, BioMerieux, France). ${ }^{12}$ Wells originally containing uninoculated medium, non-biofilm-producing bacteria and known biofilm producing bacteria were used as controls for cutoff, negative controls, and positive controls respectively. 
The tests were carried out in quadruplicate, results averaged and standard deviation calculated. To compensate for background absorbance, OD readings of the sterile medium was subtracted from all test values. The mean OD value obtained from the media control well was deducted from all the test OD values. The cut off was calculated as three standard deviations above the mean OD. ${ }^{13}$

Cut off: mean of sterile medium $+3 \mathrm{SD}$

All results were entered in an excel sheet and using SPSS 15, each isolate was classified as follows: ${ }^{14,15}$

1. Weak biofilm producer $\mathrm{OD}=<2 \mathrm{x}$ cut off

2. Moderate biofilm producer $=2 \mathrm{x}$ cut off and $<4 \mathrm{x}$ cut off,

3. Strong biofilm producer $=>4 \mathrm{x}$ cut off.

\section{Results}

\section{Control group:}

Of the 50 strains isolated from swabs taken around the catheter insertion site, 5 strains (coagulase negative staphylococcus) were weak biofilm producers at $20 \mathrm{hrs}$. incubation in tryptic soya broth with $0.25 \%$ glucose. These strains did not produce the biofilm under the remaining conditions of the study. The remaining strains were non producers.

\section{Study Group:}

Of the 326 different clinical samples, 47 were culture positive with single isolates from 44 samples and 2 isolates from each of the 3 remaining samples $(1$ suction tip and two tracheostomy tip) (Table1).
Table 1: Different indwelling medical devices $(n=326)$

\begin{tabular}{lccc}
\hline Sample & $\begin{array}{c}\text { Total } \\
\mathbf{n = 3 2 6}\end{array}$ & No growth & $\begin{array}{c}\text { Culture positive } \\
\mathbf{n = 5 0}\end{array}$ \\
\hline Catheter blood & 188 & 169 & 19 \\
Catheter urine & 30 & 29 & 1 \\
Catheter tip & 68 & 58 & 10 \\
Common bile duct stent & 10 & 5 & 5 \\
Endotracheal tip & 16 & 10 & 6 \\
Cerebro-vascular shunt & 5 & 2 & 3 \\
Suction tip & 3 & 2 & 1 \\
Tracheostomy tip & 6 & 4 & 2 \\
\hline \multicolumn{1}{c}{ Total } & $\mathbf{3 2 6}$ & $\mathbf{2 7 9}$ & $\mathbf{4 7}$ \\
\hline \multicolumn{1}{c}{}
\end{tabular}

The source and identification of these isolates is given in Table 2 .

The 50 bacterial strains isolated from indwelling devices were subjected to in-vitro biofilm production. Results of quantitative assessment of in-vitro biofilm production by different organisms are given in Table 3 and 4 respectively as producer and non-producers of biofilms. Producer strains were further classified as weak, moderate and strong producers. Biofilm productions under the different conditions of the study are given in Table 5. 
Table 2: Organisms isolated from different clinical samples. Sample

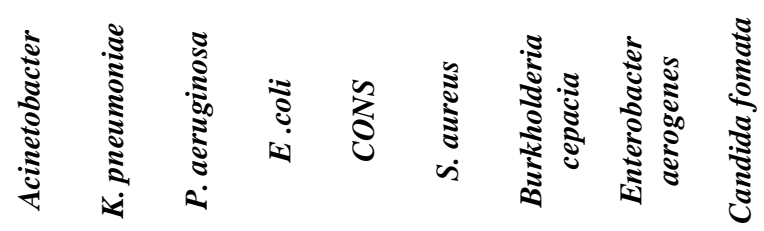

\begin{tabular}{lccccccccc}
\hline Catheter blood & 5 & 6 & 5 & 1 & 1 & 1 & 0 & 0 & 0 \\
Catheter tip & 3 & 2 & 2 & 0 & 1 & 2 & 1 & 0 & 0 \\
Catheter sample of urine & 0 & 0 & 0 & 0 & 1 & 0 & 0 & 0 & 0 \\
CBD stent & 0 & 2 & 1 & 4 & 0 & 0 & 0 & 0 & 0 \\
ET tip & 6 & 0 & 0 & 0 & 0 & 0 & 0 & 0 & 0 \\
\hline Stent & 0 & 1 & 0 & 1 & 0 & 0 & 0 & 1 & 0 \\
Suction tip & 0 & 0 & 0 & 0 & 0 & 0 & 0 & 0 & 1 \\
\hline Tracheostomy tip & 1 & 0 & 0 & 0 & 1 & 0 & 0 & 0 & 0 \\
\hline Total & $\mathbf{1 5}$ & $\mathbf{1 1}$ & $\mathbf{8}$ & $\mathbf{6}$ & $\mathbf{4}$ & $\mathbf{3}$ & $\mathbf{1}$ & $\mathbf{1}$ & $\mathbf{1}$ \\
\hline
\end{tabular}

Table 3: Quantitative analysis of biofilm production Gram positive cocci and Candida spp.

\begin{tabular}{|c|c|c|c|c|c|c|}
\hline \multirow{2}{*}{ Organism } & \multirow{2}{*}{ Total } & \multirow{2}{*}{$\begin{array}{c}\text { Non } \\
\text { producers }\end{array}$} & \multicolumn{4}{|c|}{ Producers } \\
\hline & & & Weak & Moderate & strong & Total \\
\hline$\frac{\text { Gram positive cocci }}{\text { CONS }}$ & 4 & 0 & 0 & 1 & 3 & 4 \\
\hline S. aureus & 3 & 0 & 0 & 2 & 1 & 3 \\
\hline$\frac{\text { Candida spp. }}{\text { C. fomata }}$ & 1 & 1 & 0 & 0 & 0 & 0 \\
\hline Total & 8 & 1 & $\mathbf{0}$ & 3 & 4 & 7 \\
\hline
\end{tabular}

CONS Coagulase negative staphylococci

Table 4: Quantitative analysis of biofilm production Gram negative bacilli

\begin{tabular}{|c|c|c|c|c|c|c|}
\hline \multirow{2}{*}{ Organism } & \multirow{2}{*}{ Total } & \multirow{2}{*}{$\begin{array}{c}\text { Non } \\
\text { producers }\end{array}$} & \multicolumn{4}{|c|}{ Producers } \\
\hline & & & Weak & Moderate & strong & Total \\
\hline A. baumannii & 15 & 1 & 1 & 9 & 4 & 14 \\
\hline K. pneumoniae & 11 & 1 & 1 & 1 & 8 & 10 \\
\hline P. aeruginosa & 8 & 2 & 0 & 1 & 5 & 6 \\
\hline E. coli & 6 & 3 & 1 & 0 & 2 & 3 \\
\hline B. cepacia & 1 & 0 & 0 & 0 & 1 & 1 \\
\hline E. aerogenes & 1 & 0 & 0 & 1 & 0 & 1 \\
\hline Total & 42 & 7 & 3 & 12 & 20 & 35 \\
\hline
\end{tabular}


Table 5: Biofilm production in response to different concentrations of glucose and different incubation times.

\begin{tabular}{cccccccccccc}
\hline & \multicolumn{1}{c}{ TSB } & \multicolumn{9}{c}{ TSB $(\mathbf{0 . 2 5} \%$ glucose) } & \multicolumn{3}{c}{ TSB (0.5\%glucose) } \\
\hline & $16 \mathrm{hrs}$ & $20 \mathrm{hrs}$ & $24 \mathrm{hrs}$ & $16 \mathrm{hrs}$ & $20 \mathrm{hrs}$ & $24 \mathrm{hrs}$ & $16 \mathrm{hrs}$ & $20 \mathrm{hrs}$ & $24 \mathrm{hrs}$ & Total \\
\cline { 2 - 11 } Strong & 5 & 6 & 6 & 6 & 12 & 5 & 14 & 13 & 6 & $\mathbf{7 3}$ \\
Moderate & 5 & 9 & 9 & 4 & 10 & 3 & 3 & 8 & 6 & $\mathbf{5 7}$ \\
Weak & 10 & 7 & 9 & 13 & 10 & 7 & 5 & 7 & 9 & $\mathbf{7 7}$ \\
\hline Total & $\mathbf{2 0}$ & $\mathbf{2 2}$ & $\mathbf{2 4}$ & $\mathbf{2 3}$ & $\mathbf{3 2}$ & $\mathbf{1 5}$ & $\mathbf{2 2}$ & $\mathbf{2 8}$ & $\mathbf{2 1}$ & \\
\hline
\end{tabular}

\section{Discussion:}

Indwelling medical devices are frequently used in all health setting, with critical care units of hospitals often using multiple medical devices for treatment and intervention in patient care.

Of the 50 samples tested for biofilm production, the commonest sample $(n=19 ; 38 \%)$ was blood obtained through a catheter. Peripheral vein sampling was done to rule out general bacteremia in all these patients to rule out seeding of the catheter through the blood. Blood catheter tips contributed to $20 \%$ (11/50). Intravascular catheters may be inserted for administration of fluids, blood products, medications, nutritional solutions, and hemodynamic monitoring. These organisms may originate from the skin of patients or healthcare workers, to which entry ports are exposed, or other sources in the environment. ${ }^{2}$

Acinetobacter spp., Pseudomonas spp., Klebsiella spp., Staphylococcus spp., and E. coli are the commonest causes of nosocomial infections. Colonization of indwelling medical devices with consequent biofilm production is a likely contributory factor to such infections. These microorganisms survive in the hospital environment despite unfavorable conditions such as desiccation, nutrient starvation, and antimicrobial treatment. It is hypothesized that the ability of these microbes to persist in these environments, as well as their virulence may be a result of their capacity to colonize medical devices. ${ }^{16}$

In a study conducted by Summaiya Mulla et al. ${ }^{17}$ it was documented that overall biofilm production from bacterial isolates from patients having medical devices was $88 \%$ which is similar to the results of the current study where 42 of $50(84 \%)$ strains were biofilm producers in contrast to the $20 \%$ production by surface colonizers. The most common isolates which produced biofilm in their study was $P$. aeruginosa (23/100 strains), Acinetobacter spp.(23/100 strains) followed by $K$. pneumoniae (20/100 strains). In contrast, the highest production of biofilm in the current study was detected in Acinetobacter spp.(15/50), followed by Klebsiella spp.(11/50) and Pseudomonas spp.(8/50). A study by Donlanet al., ${ }^{2}$ showed that the organisms most commonly isolated from central venous catheter biofilms were S. epidermidis, $S$. aureus, $C$. albicans, $P$. aeruginosa, K. pneumoniae, and E. faecalis. ${ }^{2,18,19}$

Twenty hours incubation was found to be optimum for detection of biofilms produced by bacteria in the current study. Bacteria produced more biofilm in $0.25 \%$ concentration of glucose in tryptic soya broth when compared to other concentrations. The observation in our study was that strong biofilm production by the isolates persisted even up to 24 hours, whereas with weak and moderate biofilm producers, production was reduced at 20 hours of incubation. 


\section{Conclusions:}

The current study shows that biofilm producing Gram negative bacilli are present in vascular catheters. Methods that detect and quantify biofilms on the inner (luminal) as well as outer surfaces of catheters will provide the only true picture of biofilm colonization. More reliable quantitative methods include the use of mechanical forces (e.g., sonication or vortexing) to remove the biofilm associated organisms, which can then be quantified by means of plate count or fluorescent staining techniques. ${ }^{18}$

Further studies are needed to assess both risks to the patient and implementable infection control procedures to reduce such risks.

Acknowledgement: The Gujarat Cancer Society for funding the study.

\section{References:}

1. $\quad$ Donlan RM. Biofilms: Microbial life on surfaces. Emerg. Infect. Dis. 2002; 8:881890. http://dx.doi.org/10.3201/eid0809.020063

2. Donlan RM. Biofilms and device-associated infections. Emerg. Infect. Dis. 2001; 7:277-281. http://dx.doi.org/10.3201/eid0702.010226

3. Stoodley P., Sauer K., Davies DG., Costerton JW. Biofilms as complex differentiated communities. Annu. Rev. Microbiol. 2002; 56:187-209.

4. Fey PD., Olson ME. Current concepts in biofilm formation of Staphylococcus epidermidis. Future Microbiol. 2010; 5:917-933. http://dx.doi.org/10.1146/annurev.micro.56.012302.160705

5. Costerton JW., Stewart PS., Greenberg EP. Bacterial biofilms: A common cause of persistent infections. Science 1999; 284:1318-1322.

http://dx.doi.org/10.1126/science.284.5418.1318

6. Fux CA., Costerton JW., Stewart PS., Stoodley P. Survival strategies of infectious biofilms. Trends Microbiol. 2005; 13:34-40. http://dx.doi.org/10.1016/j.tim.2004.11.010

7. Stewart PS., Costerton JW. Antibiotic resistance of bacteria in biofilms. Lancet 2001; 358:135-138. http://dx.doi.org/10.1016/S0140-6736(01)05321-1

8. Otto M. Staphylococcal biofilms. Curr.Top.Microbiol.Immunol. 2008; 322:207228. No doi

9. Hola V., Ruzicka F., Votava M. The dynamics of Staphylococcus epidermis biofilm formation in relation to nutrition, temperature and time. Scripta Medica 2006; 79:169-74.

No doi

10. Raad II., Sabbagh MF., Rand KH., Sherertz RJ. Quantitative tip culture methods and the diagnosis of central venous. Catheter-related infections. Diagn Microbiol Infect Dis 1992; 15:13-20. No doi

11. Rao RS., Karthika RU., Singh SP., et al. Correlation between biofilm production and multiple drug resistance in imipenem resistant clinical isolates of Acinetobacter baumannii. Indian J Med Microbiol 2008; 26:333-7. 
http://dx.doi.org/10.4103/0255-0857.43566

12. Eftekhar F., Mirmohamadi Z.. Evaluation of biofilm production by Staphylococcus epidermidis isolates from nosocomial infections and skin of healthy volunteers. Int J Med Med Sci 2009; 1:438-41. No doi

13. Christensen G., Simpson W., Younger J., et al. Adherence of coagulase-negative staphylococci to plastic tissue culture plates: A quantitative model for the adherence of staphylococci to medical devices. J Clin Microbiol 1985; 22:9961006. No doi

14. Jayanthi M., Ananthasubramanian M., Appalaraju B. Assessment of Pheromone response in biofilm forming clinical isolates of high level gentamicin resistant Enterococcus faecalis. Indian J Med Microbiol 2008; 26:248-51.

http://dx.doi.org/10.4103/0255-0857.42037

15. Summaiy Mulla., Sangia Revdiwala. Assessment of biofilm formation in deviceassociated clinical bacterial isolates in tertiary level hospital. Indian Journal Pathology Microbiology 2011; 54:561-4. http://dx.doi.org/10.4103/0377-4929.85093

16. Gelosia A., Baldassarri L., Deighton M., van Nguyen T. Phenotypic and genotypic markers of Staphylococcus epidermidis virulence. Clin Microbiol Infect 2001; 7:193-9. http://dx.doi.org/10.1046/j.1469-0691.2001.00214.x

17. Stickler DJ. Bacterial biofilms and the encrustation of urethral catheters.

Biofouling 1996; 94:293-305. http://dx.doi.org/10.1080/08927019609378311

18. Donlan M. Biofilm formation : A clinically relevant microbiological process. Clin Infect Dis 2001; 33(8):1387-92 http://dx.doi.org/10.1086/322972 